\title{
Exploratory lattice QCD study of the rare kaon decay $K^{+} \rightarrow \pi^{+} v \bar{v}$
}

\author{
Norman H. Christ ${ }^{a}$, Xu Feng ${ }^{*}$, Andreas Jüttner ${ }^{b}$, Andrew Lawson ${ }^{b}$, Antonin \\ Portelli ${ }^{b}$, Christopher T. Sachrajda ${ }^{b}$ \\ a Physics Department, Columbia University, New York, NY 10027, USA \\ ${ }^{b}$ School of Physics and Astronomy, University of Southampton, Southampton SO17 1BJ, UK \\ E-mail: nhc@phys.columbia.edu, pkufengxu@gmail.com, \\ juettner@soton.ac.uk, allg13@soton.ac.uk, antonin.portelli@me.com, \\ ctsesoton.ac.uk
}

As flavor changing neutral current processes, rare kaon decays $K \rightarrow \pi v \bar{v}$ are highly suppressed in the standard model and thus provide ideal probes for the observation of new physics effects. Given the importance of rare kaon decays, the CERN NA62 experiment aims at an observation of $O(100)$ events of $K^{+} \rightarrow \pi^{+} v \bar{v}$ decay and a $10 \%$-precision measurement of the branching ratio in two years. Another experiment, J-PARC KOTO, is dedicated to search for the CP-violating $K_{L} \rightarrow \pi^{0} v \bar{v}$ decay. Recognizing that the standard model predictions will soon be confronted with new experimental results, it is important to determine the long-distance contributions to $K^{+} \rightarrow \pi^{+} v \bar{v}$ with a controlled error. Here I will present an exploratory calculation of the longdistance contributions to the $K^{+} \rightarrow \pi^{+} v \bar{v}$ decay amplitude from first principles using lattice QCD.

The 8th International Workshop on Chiral Dynamics, CD2015 ***

29 June 2015 - 03 July 2015

Pisa, Italy

\footnotetext{
* Speaker.
} 


\section{Introduction}

Rare kaon decays have attracted increasing interest during the past few decades. As flavor changing neutral current processes, these decays are highly suppressed in the standard model (SM) and thus provide ideal probes for the observation of new physics effects. The $K \rightarrow \pi \nu \bar{v}$ decay is particularly interesting because it is known to be short-distance (SD) dominated and theoretically clean. The required hadronic matrix elements can be obtained from leading order semi-leptonic $K$ decays, such as $K^{+} \rightarrow \pi^{0} e^{+} v$, via isospin rotation. The remaining long-distance (LD) contributions below the charm scale are safely neglected in $K_{L} \rightarrow \pi^{0} v \bar{v}$ and are expected to be small in $K^{+} \rightarrow$ $\pi^{+} v \bar{v}$. Though small, by including the LD contribution estimated by a phenomenological analysis, which involves chiral perturbation theory and the operator production expansion, the branching ratio $\operatorname{Br}\left(K^{+} \rightarrow \pi^{+} v \bar{v}\right)$ is enhanced by $6 \%$ [1], which is comparable to the 8-10\% total SM error [2].

The current known measurement of the branching ratio [3] is a combined result based on the 7 events collected by BNL E787 [4, 5, 6, 7] and its successor E949 [8, 3]. Its central value is almost twice of the SM prediction, but with a $60-70 \%$ uncertainty it is still consistent with SM. The new experiment, NA62 at CERN [9, 10], aims at an observation of $O(100)$ events and a 10\%precision measurement of $\operatorname{Br}\left(K^{+} \rightarrow \pi^{+} v \bar{v}\right)$ in two years. Another new experiment, KOTO at J-PARC [11, 12], is designed to search for CP-violating $K_{L} \rightarrow \pi^{0} v \bar{v}$ decay, and may have good chance to find the events. Considering the fact that the SM predictions will be confronted with the new experiment soon, a lattice QCD calculation of the LD contribution to $K^{+} \rightarrow \pi^{+} v \bar{v}$ is important.

It has been proposed to calculate the LD contributions to $K \rightarrow \pi v \bar{v}$ using lattice QCD in Ref. [13]. The method to treat with a second-order electroweak amplitude on the lattice has been developed by Refs. $[14,15]$ and applied to the lattice calculation of the LD contributions to $K_{L^{-}}$ $K_{S}$ mass difference [16, 17], the indirect CP violation parameter $\varepsilon_{K}[18]$ and the rare kaon decay $K \rightarrow \pi \ell^{+} \ell^{-}[19,20,21]$. Adopting the useful techniques provided by the previous studies, we have developed a lattice method to calculate the decay amplitude for $K \rightarrow \pi v \bar{v}$. In this work we will report a lattice QCD study on rare kaon decay $K^{+} \rightarrow \pi^{+} v \bar{v}$ at a heavier-than-physical pion mass $m_{\pi}=420 \mathrm{MeV}$. Our exploratory study involves the calculation of both $W-W$ and $Z$-exchange diagrams.

\section{Contributions from intermediate quark loops}

In the SM the $K \rightarrow \pi v \bar{v}$ decays proceed through $W-W$ and $Z$-exchange diagrams, which contain three intermediate up-type quark loops. A rough estimate reflecting the quadratic GIM mechanism gives a comparison among the top, charm and up quark contribution to the decay amplitude [22]

$$
\lambda_{t} x_{t}: \lambda_{c} x_{c} \ln x_{c}: \lambda_{u} \frac{\Lambda_{\mathrm{QCD}}^{2}}{M_{W}^{2}},
$$

where $x_{q}=m_{q}^{2} / M_{W}^{2}$ for $q=u, c, t$. The relevant CKM factors are defined as $\lambda_{q}=V_{q s}^{*} V_{q d}$. In Eq. $(2.1)$ the largest contribution to the decay amplitude $(\sim 68 \%)$ comes from the top quark loop. The charm quark contribution is suppressed by a factor of $x_{c} \ln x_{c} / x_{t}$ but compensated with another 
factor $\lambda_{c} / \lambda_{t}$ from the CKM matrix element. So it still accounts for $\sim 29 \%$ of the total contribution. The remaining up quark contribution is estimated to be $\sim 3 \%$.

Among the three up-type quarks, the top quark contribution is a SD contribution while the up quark contribution is a LD contribution. The charm quark contribution carries a logarithmic factor $\ln x_{c}$ as shown in Eq. (2.1). This logarithmic structure suggests that the contribution comes from a high energy scale $\sim M_{W}$ down to an energy scale of the charm quark mass $\sim m_{c}$ and thus is SD dominated. The SD part of the charm quark contribution can be calculated precisely using perturbation theory $[23,24]$. Our concern here is how to calculate the LD part of the charm quark contribution, as well as the up quark contribution, with controlled error.

\section{Bilocal operators}

Instead of integrating out the charm quark field, our calculation is performed in the fourflavor theory. This allows us to explore the bilocal structure for up and charm quark contributions directly. We can begin with the first-order effective field theory, where the $W$ and $Z$ bosons have been integrated out. In the $W$-W diagrams the $W$-boson exchanges have been replaced by two effective operators

$$
\begin{aligned}
& O_{q \ell}^{\Delta S=1}=C_{\Delta S=1}^{\overline{\mathrm{MS}}}(\mu)\left[(\bar{s} q)_{V-A}(\bar{v} \ell)_{V-A}\right]^{\overline{\mathrm{MS}}}(\mu), \\
& O_{q \ell}^{\Delta S=0}=C_{\Delta S=0}^{\overline{\mathrm{MS}}}(\mu)\left[(\bar{\ell} v)_{V-A}(\bar{q} d)_{V-A}\right]^{\overline{\mathrm{MS}}}(\mu) .
\end{aligned}
$$

The Wilson coefficients $C_{\Delta S=1}^{\overline{\mathrm{MS}}}(\mu)$ and $C_{\Delta S=0}^{\overline{\mathrm{MS}}}(\mu)$ are calculated in the $\overline{\mathrm{MS}}$ scheme and account for the contributions from the SD physics. Since the hadronic part of $O_{q \ell}^{\Delta S=1}$ and $O_{q \ell}^{\Delta S=0}$ is given by a $V-A$ current, $C_{\Delta S=1}^{\overline{\mathrm{MS}}}(\mu)=C_{\Delta S=0}^{\overline{\mathrm{MS}}}(\mu)=1$. The operators $(\bar{s} q)_{V-A}(\bar{v} \ell)_{V-A}$ and $(\bar{\ell} v)_{V-A}(\bar{q} d)_{V-A}$ have been renormalized in the $\overline{\mathrm{MS}}$ scheme at the scale $\mu$. Given a $\overline{\mathrm{MS}}$ operator $O^{\overline{\mathrm{MS}}}(\mu)$, it can be related to the bare lattice operator $O^{\text {lat }}(a)$ through the relation $O^{\overline{\mathrm{MS}}}(\mu)=Z_{O}^{\text {lat } \rightarrow \overline{\mathrm{MS}}}(\mu, a) O^{\text {lat }}(a)$, where $Z_{O}^{\text {lat } \rightarrow \overline{\mathrm{MS}}}(\mu, a)$ is a conversion factor and $a$ is the lattice spacing.

In the $Z$-exchange diagrams the $W$-boson exchange has been described by a $O_{q}^{W}$ four-quark operator

$$
O_{q}^{W}=C_{1}^{\overline{\mathrm{MS}}}(\mu) Q_{1, q}^{\overline{\mathrm{MS}}}(\mu)+C_{2}^{\overline{\mathrm{MS}}}(\mu) Q_{2, q}^{\overline{\mathrm{MS}}}(\mu)
$$

where $Q_{i, q}^{\overline{\mathrm{MS}}}(\mu)(i=1,2)$ are conventional current-current operators renormalized in the $\overline{\mathrm{MS}}$ scheme. They can be related to the bare lattice operators through

$$
\begin{aligned}
& Q_{i, q}^{\overline{\mathrm{MS}}}(\mu)=\sum_{j} Z_{i, j}^{\mathrm{lat} \rightarrow \overline{\mathrm{MS}}}(\mu) Q_{j, q}^{\mathrm{lat}}, \quad j=1,2 \\
& Q_{1, q}^{\mathrm{lat}}=\left(\bar{s}_{a} q_{b}\right)_{V-A}\left(\bar{q}_{b} d_{a}\right)_{V-A}, \quad Q_{2, q}^{\mathrm{lat}}=\left(\bar{s}_{a} q_{a}\right)_{V-A}\left(\bar{q}_{b} d_{b}\right)_{V-A}
\end{aligned}
$$

where $a, b$ are color indices. The $Z$-boson exchange has been replaced by a two-quark/two-neutrino operator $O_{\ell}^{Z}$

$$
O_{\ell}^{Z}=C_{Z}^{\overline{\mathrm{MS}}}(\mu)\left[J_{\mu}^{Z} \bar{v}_{\ell} \gamma^{\mu}\left(1-\gamma_{5}\right) v_{\ell}\right]^{\overline{\mathrm{MS}}}(\mu)
$$


where the neutral current $J_{\mu}^{Z}$ is given by $J_{\mu}^{Z}=\sum_{q=u, c, d, s} T_{3}^{q} \bar{q} \gamma_{\mu}\left(1-\gamma_{5}\right) q-2 Q_{e m, q} \sin ^{2} \theta_{W} \bar{q} \gamma_{\mu} q$. The weak isospin factor $T_{3}^{q}$ takes the values $+1 / 2,+1 / 2,-1 / 2,-1 / 2$ for $q=u, c, d, s$ and the electromagnetic charge $Q_{e m, q}$ is given by $2 / 3$ for up-type quarks and $-1 / 3$ for down-type quarks. As described above, we have $C_{Z}^{\overline{\mathrm{MS}}}(\mu)=1$.

Given the effective operators, which represent the first-order weak interaction, we can construct the bilocal operators for the second-order interaction

$$
\begin{aligned}
\mathscr{B}_{W W}(y) & =\int d^{4} x T\left[O_{u \ell}^{\Delta S=1}(x) O_{u \ell}^{\Delta S=0}(y)\right]-\{u \rightarrow c\} \\
\mathscr{B}_{Z}(y) & =\int d^{4} x T\left[O_{u}^{W}(x) O_{\ell}^{Z}(y)\right]-\{u \rightarrow c\} .
\end{aligned}
$$

Here $\mathscr{B}_{W W}(y)$ and $\mathscr{B}_{Z}(y)$ are the bilocal operators associated with $W$ - $W$ diagrams and $Z$-exchange diagrams, respectively. The minus sign in Eqs. (3.5a) and (3.5b) indicates the GIM cancellation between the intermediate up and charm quark contributions.

Note that in lattice QCD calculations, the bilocal operator $\mathscr{B}_{W W}$ are $\mathscr{B}_{Z}(y)$ are constructed using the bare lattice operators. The conversion factor $Z_{O}^{\text {lat } \rightarrow \overline{\mathrm{MS}}}(\mu, a)$ has removed the lattice spacing dependence in the bare lattice operator. However, when two lattice operators approach to each other, a new lattice spacing dependence could possibly appear. In our study of rare kaon decay, due to the logarithmic SD divergence in $\mathscr{B}_{W W}$ and $\mathscr{B}_{Z}$, we need to introduce a counter term to remove this new lattice spacing dependence. Namely we calculate the $K^{+} \rightarrow \pi^{+} v \bar{v}$ decay amplitude by evaluating matrix elements of

$$
\left\langle\pi^{+} v \bar{v}\left|\mathscr{B}_{W W}(0)+\mathscr{B}_{Z}(0)\right| K^{+}\right\rangle+\left\langle\pi^{+} v \bar{v}\left|C_{W}(a) O^{(6), \text { lat }}(0)\right| K^{+}\right\rangle
$$

where $C_{W}(a) O^{(6), \text { lat }}(0)$ is a counter term, which also contains the usual SD contributions. The

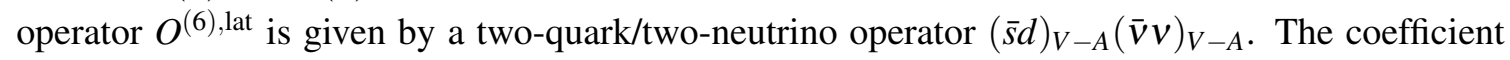
$C_{W}(a)$ can be determined by exploiting the non-perturbative Rome-Southampton methods [25, 26, 27] and electro-weak/QCD perturbation theory.

\section{Research program}

Using the methods described in the above sections, we can compute the LD contributions to the rare kaon decay amplitudes. As the first step we use an ensemble with the lattice size $(L / a)^{3} \times(T / a)=16^{3} \times 32$, the pion mass $m_{\pi}=420 \mathrm{MeV}$, the kaon mass $m_{K}=540 \mathrm{MeV}$, the charm quark mass $m_{c}^{\overline{\mathrm{MS}}}(2 \mathrm{GeV})=860 \mathrm{MeV}$, and an inverse lattice spacing $a^{-1}=1.73 \mathrm{GeV}$. Although the parameters are unphysical, the small lattice significantly reduces the requirement on computer resources and allows us to set up the calculation and test our methods. Our calculation involves both $W-W$ and $Z$-exchange diagrams. The statistical error of the LD contribution to the rare kaon decay amplitude can be reduced to few-percent level by measuring 800 configurations.

In the study on the $16^{3} \times 32$ lattice, the systematic errors are not under control. One of the significant systematic effects is from the $420 \mathrm{MeV}$ pion mass. Approaching the physical pion mass requires a larger lattice volume. Therefore, as the second step, we are performing the calculation using an ensemble with a near-physical pion mass $m_{\pi}=170 \mathrm{MeV}$ and a larger volume $32^{3} \times 64$ (lattice spacing inverse $a^{-1}=1.37 \mathrm{GeV}$ ). The second-step calculation allows us to achieve better 
control of the systematic effects from the unphysical pion mass. By using the lighter pion mass, the allowed momentum region for $\left\{p_{\pi}, p_{v}, p_{\bar{v}}\right\}$ in the $K \rightarrow \pi v \bar{v}$ decay increases and we need to evaluate the decay amplitude for multiple momenta. In addition, for this $32^{3} \times 64$ calculation, the mass of the kaon is larger than the energy of the two-pion state. Thus the exponentially growing contamination from the two-pion intermediate state needs to be accounted for properly.

Another significant systematic effect arises from the lighter-than-physical charm quark mass. Including the physical charm quark mass would require an ultra-fine lattice spacing. We plan to use an ensemble with a lattice volume $64^{3} \times 128$, including both physical pion and charm quark masses. Here the lattice spacing $a^{-1}=2.38 \mathrm{GeV}$ is not sufficiently fine and will still cause a large lattice artifact $\sim 20 \%$. However, the LD contributions to the rare kaon decay amplitude are much smaller than the SD top and charm quark contributions. Thus we expect that this lattice artifact will only cause $\mathrm{a} \sim 1 \%$ uncertainty in the total branching ratio.

\section{Numerical results for $W-W$ diagrams}

In this section we present our numerical results for $W-W$ diagrams. We first introduce the scalar amplitude which can be extracted from the bilocal matrix element.

\subsection{Scalar amplitude}

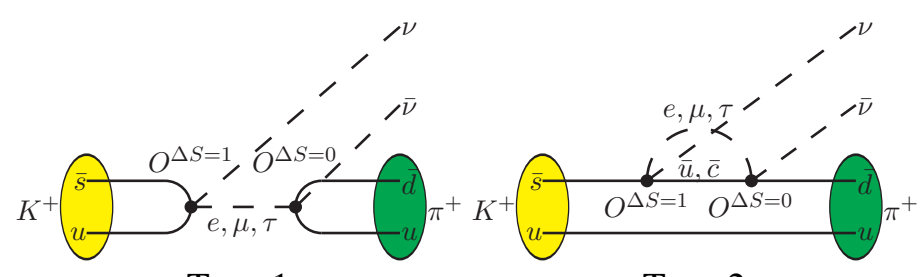

Type 1

Type 2

Figure 1: Quark and lepton contractions for $W-W$ diagrams.

In $W$-W diagrams, using the bilocal operator $\mathscr{B}_{W W}$, we can calculate the matrix element

$$
T_{W W}=\int d^{4} x\left\langle\pi^{+} v \bar{v}\left|T\left\{O_{u \ell}^{\Delta S=1}(x) O_{u \ell}^{\Delta S=0}(0)\right\}\right| K^{+}\right\rangle-\{u \rightarrow c\},
$$

where the operators $O_{u \ell}^{\Delta S=1}$ and $O_{u \ell}^{\Delta S=0}$ are defined in Eq. (3.1). The quark and lepton contractions for $T_{W W}$ are shown in Fig. 1.

Due to the chiral property of the massless neutrino and anti-neutrino, we can write $T_{W W}$ as a combination of the scalar amplitude $F_{W W}\left(p_{K}, p_{v}, p_{\bar{v}}\right)$ and a spinor product $\bar{u}\left(p_{v}\right) p_{K}\left(1-\gamma_{5}\right) v\left(p_{\bar{v}}\right)$ through

$$
T_{W W}=(+i) F_{W W}\left(p_{K}, p_{v}, p_{\bar{v}}\right)\left[\bar{u}\left(p_{v}\right) p_{K}\left(1-\gamma_{5}\right) v\left(p_{\bar{v}}\right)\right],
$$

where $p_{K}, p_{v}$ and $p_{\bar{v}}$ are the momenta for kaon, neutrino and anti-neutrino, respectively. In the sections which describe the numerical results, we use Euclidean conventions for $\gamma$-matrices and momenta. The $+i$ factor appears due to the usage of Euclidean $\gamma$-matrices. For an on-shell particle with mass $m$, the four momentum using Euclidean conventions is given by $p=(\vec{p}, i E)$, where $E=\sqrt{m^{2}+\vec{p}^{2}}$. 


\subsection{Dalitz plot}

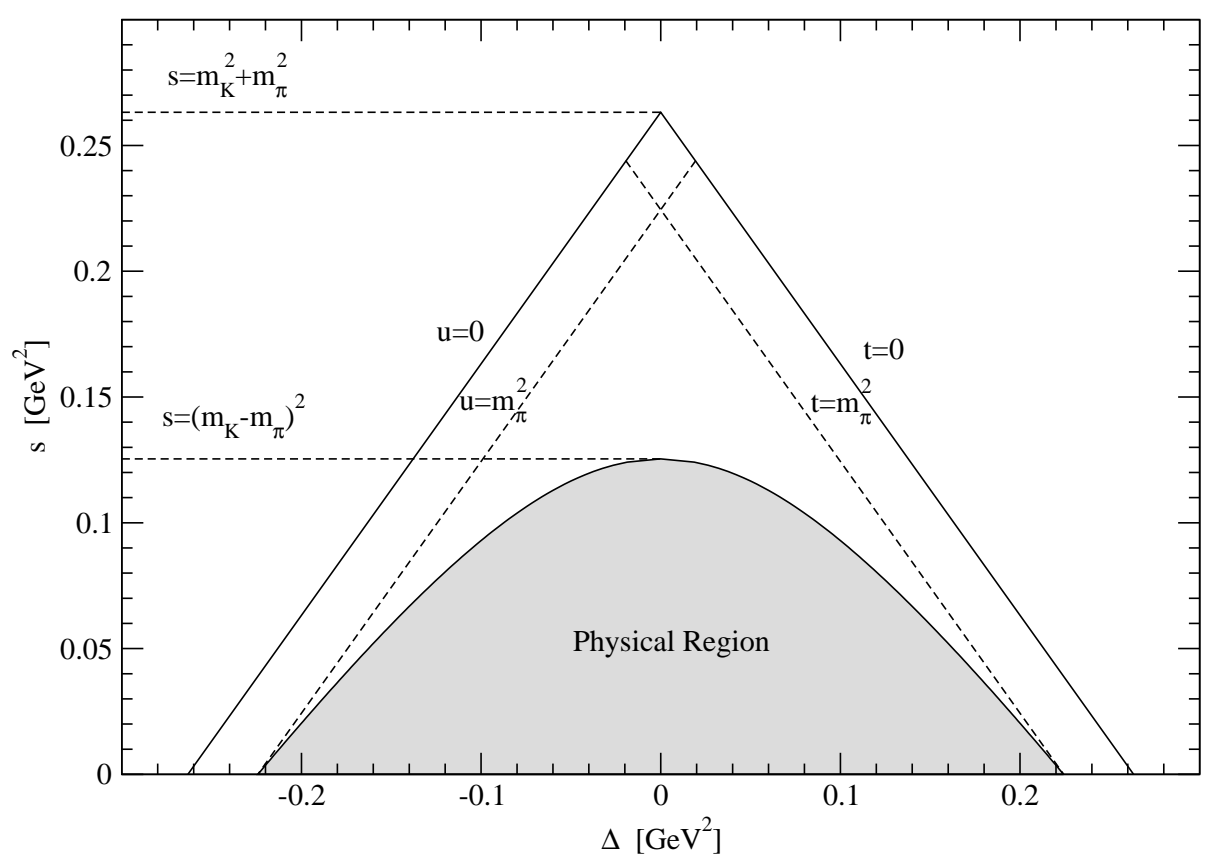

Figure 2: Dalitz plot for $K \rightarrow \pi \nu \bar{v}$.

Given the momenta $p_{K}, p_{\pi}, p_{v}$ and $p_{\bar{v}}$, one can define three Lorentz invariants

$$
s=-\left(p_{K}-p_{\pi}\right)^{2}, \quad t=-\left(p_{K}-p_{v}\right)^{2}, \quad u=-\left(p_{K}-p_{\bar{v}}\right)^{2},
$$

where two of them are independent: $s+t+u=m_{K}^{2}+m_{\pi}^{2}$. The physical region for these invariants is shown in the Dalitz plot of Fig. 2, where we use the invariant $s$ as the $y$-axis and $\Delta=u-t$ as the $x$-axis. Using the Dirac equation for the massless neutrinos, one can show that the modulus of the decay amplitude vanishes at the edge of the Dalitz plot. Therefore, we are more interested in momenta that are far away from the edge. Note that in the $K \rightarrow \pi v \bar{v}$ decay it is almost impossible to measure the neutrino momenta in today's experiments. Once we obtain the $(\Delta, s)$ dependence of the decay amplitude, we need to integrate over the parameter $\Delta$ and obtain a differential decay rate $d \Gamma / d s$, which can be measured directly by experiments.

On the $16^{3} \times 32$ lattice with a $420 \mathrm{MeV}$ pion mass, we studied the decay amplitude at a single momentum $(\Delta, s)=(0,0)$. For this momentum the neutrino and the anti-neutrino carry the same spatial momentum and the pion moves in the opposite direction with twice the momentum of each of the $v$ and $\bar{v}$. Due to the heavy pion mass used in our $16^{3} \times 32$ study, the allowed momenta for the final-state particles are constrained to lie in a narrow region. Since multiple momentum values would be so close to each other, they would be highly correlated and it would be difficult to extract a clear momentum dependence from them. For this reason, we did not examine other momentum values. The situation may change once we perform the calculation at the physical pion mass. In this case we need to calculate the scalar amplitude at various values of $(\Delta, s)$ to gain a better understanding of the momentum dependence. 


\subsection{Exponentially growing contamination at large Euclidean time}

In the second-order weak interaction, given a bilocal matrix element $\int d^{4} x\left\langle f\left|T\left[O_{1}(x) O_{2}(0)\right]\right| i\right\rangle$, one can insert a complete set of intermediate states in-between the two interpolating operators, $O_{1}$ and $O_{2}$. A Euclidean time integral in a interval of $-T_{a}<t<T_{b}\left(T_{a}, T_{b}>0\right)$ yields

$$
\begin{aligned}
& \int_{-T_{a}}^{T_{b}} d t \int d^{3} \vec{x}\left\langle f\left|T\left[O_{1}(\vec{x}, t) O_{2}(0)\right]\right| i\right\rangle \\
= & \sum_{m} \frac{\left\langle f\left|O_{1}\right| m\right\rangle\left\langle m\left|O_{2}\right| i\right\rangle}{E_{m}-E_{f}}\left(1-e^{\left(E_{f}-E_{m}\right) T_{b}}\right)+\sum_{n} \frac{\left\langle f\left|O_{2}\right| n\right\rangle\left\langle n\left|O_{1}\right| i\right\rangle}{E_{n}-E_{i}}\left(1-e^{\left(E_{i}-E_{n}\right) T_{a}}\right) \\
= & \sum_{m} \frac{\left\langle f\left|O_{1}\right| m\right\rangle\left\langle m\left|O_{2}\right| i\right\rangle}{E_{m}-E_{f}}+\sum_{n} \frac{\left\langle f\left|O_{2}\right| n\right\rangle\left\langle n\left|O_{1}\right| i\right\rangle}{E_{n}-E_{i}}+\text { exponential terms. }
\end{aligned}
$$

The third line of Eq. (5.4) gives the second-order weak matrix element together with the unwanted exponential terms. If the energies of the intermediate states is larger than that of the initial/final state, i.e. $E_{m / n}>E_{i}=E_{f}$, then these extra terms exponentially vanish at large $T_{a / b}$. On the other hand, if the energies of the intermediate states are below the energy of initial/final state, then one needs to remove these extra terms which increase exponentially at large $T_{a / b}$.

Instead of fixing the operator $O_{2}$ at time $t_{2}=0$, one can adopt the method proposed in Ref. [16] and vary the time locations of both $O_{1}$ and $O_{2}$ and sum these times over an interval of $t_{a} \leq t_{1,2} \leq t_{b}$

$$
\begin{aligned}
& \sum_{t_{1}=t_{a}}^{t_{b}} \sum_{t_{2}=t_{a}}^{t_{b}} \int d^{3} \vec{x}\left\langle\phi_{f}\left(t_{f}\right) T\left[O_{1}\left(\vec{x}, t_{1}\right) O_{2}\left(\overrightarrow{0}, t_{2}\right)\right] \phi_{i}^{\dagger}\left(t_{i}\right)\right\rangle e^{E_{i / f}\left(t_{f}-t_{i}\right)} \\
= & T_{b o x}\left\langle f\left|O_{1}(0) O_{2}(0)\right| i\right\rangle \\
+ & \sum_{m} \frac{\left\langle f\left|O_{1}\right| m\right\rangle\left\langle m\left|O_{2}\right| i\right\rangle}{E_{m}-E_{f}}\left(T_{\mathrm{box}}+\frac{e^{\left(E_{f}-E_{m}\right) T_{\mathrm{box}}}-1}{E_{m}-E_{f}}\right) \\
+ & \sum_{n} \frac{\left\langle f\left|O_{2}\right| n\right\rangle\left\langle n\left|O_{1}\right| i\right\rangle}{E_{n}-E_{f}}\left(T_{\mathrm{box}}+\frac{e^{\left(E_{f}-E_{n}\right) T_{\mathrm{box}}}-1}{E_{n}-E_{f}}\right)
\end{aligned}
$$

where $\phi_{i}^{\dagger}$ and $\phi_{f}$ are the interpolating operators that create the initial and final states. The time locations $t_{i}$ and $t_{f}$ are required to satisfy $t_{i} \ll t_{a}$ and $t_{f} \gg t_{b}$ to gaurantee the ground-state dominance. In Eq. (5.5) the time integral has been replaced by the time summation. The interval size $T_{\text {box }}$ takes the value $T_{\mathrm{box}}=t_{b}-t_{a}+a$ with $a$ the lattice spacing. The exponential terms still exist in Eq. (5.5). By evaluating the matrix elements for these intermediate states, whose energies are smaller than $E_{i}=E_{f}$, one can remove the exponentially growing contamination associated with the factor $\frac{e^{\left(E_{f / i}-E_{m / n}\right) f_{\mathrm{box}}-1}}{E_{m / n}-E_{f / i}}$. Then one can fit the $T_{\mathrm{box}}$ dependence of the integrated matrix element to a linear function $b_{0}+b_{1} T_{\text {box }}$. The slope $b_{1}$ gives the physical bilocal matrix element without exponential contamination.

\subsection{Preliminary numerical results}

To study the exponentially growing time dependence in the $W-W$ diagram, we define the unintegrated scalar amplitude with explicit $t=t_{\Delta S=1}-t_{\Delta S=0}$ time dependence, where $t_{\Delta S=1}$ is the time 
for two-quark/two-lepton operator $O_{q \ell}^{\Delta S=1}$ and $t_{\Delta S=0}$ the time for $O_{q \ell}^{\Delta S=0}$

$$
\begin{aligned}
& (+i) F_{W W}(t)\left[\bar{u}\left(p_{v}\right) \not p_{K}\left(1-\gamma_{5}\right) v\left(p_{\bar{v}}\right)\right] \\
\equiv & \int d^{3} \vec{x}\left\langle\pi^{+} v \bar{v}\left|T\left\{O_{u \ell}^{\Delta S=1}\left(\vec{x}, t_{\Delta S=1}\right) O_{u \ell}^{\Delta S=0}\left(\overrightarrow{0}, t_{\Delta S=0}\right)\right\}\right| K^{+}\right\rangle-\{u \rightarrow c\} .
\end{aligned}
$$

We have imposed the energy conservation condition $E_{K}=E_{\pi}+E_{V}+E_{\bar{v}}$ for the initial and final states. According to time translation invariance, the amplitude $F_{W W}(t)$ depends only on the time separation between $O_{u \ell}^{\Delta S=1}$ and $O_{u \ell}^{\Delta S=0}$. The scalar amplitude $F_{W W}\left(p_{K}, p_{v}, p_{\bar{v}}\right)$ can be obtained by integrating $F_{W W}(t)$ over the time separation $t$.
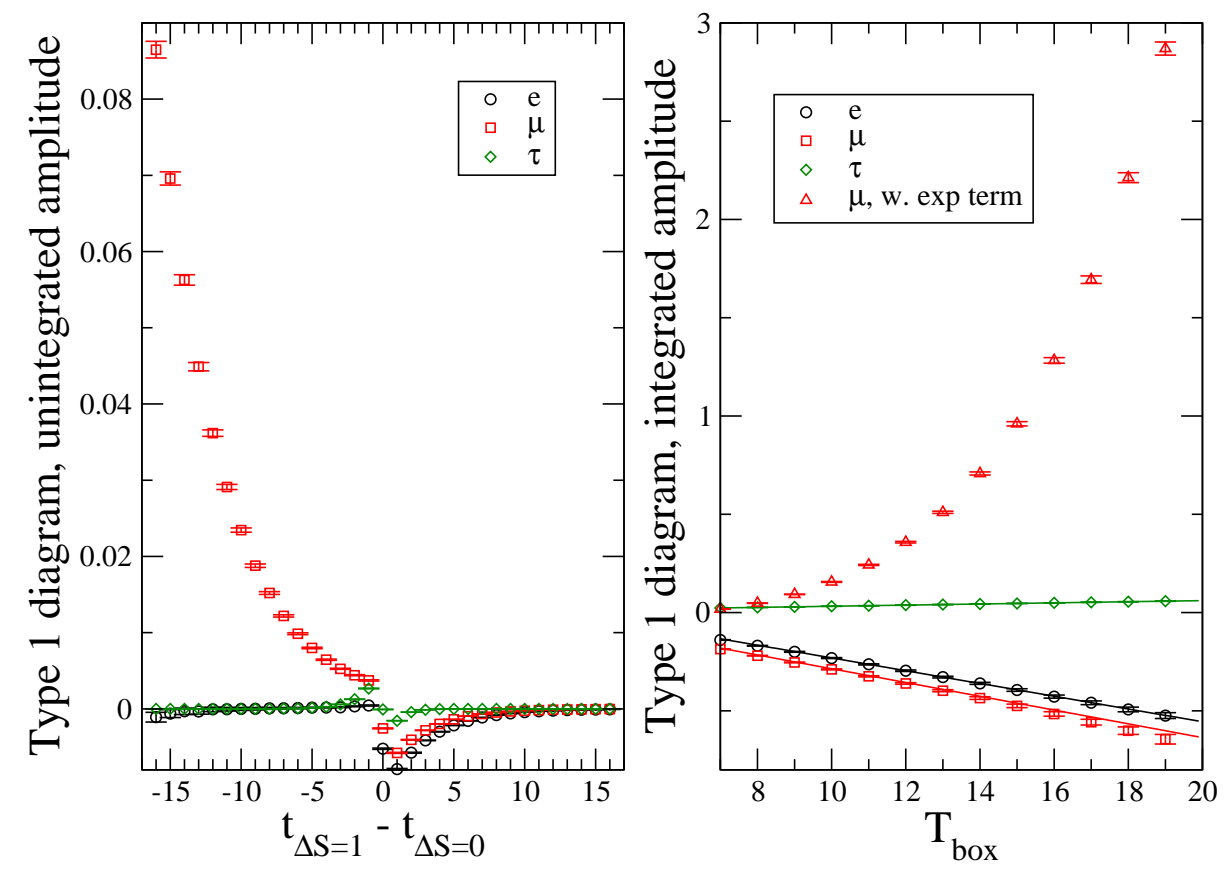

Figure 3: The scalar amplitude for Type 1 diagram. In the left panel the unintegrated scalar amplitude $F_{W W}(t)$ as a function of $t=t_{\Delta S=1}-t_{\Delta S=0}$ is shown. The black circle, red square and green diamond symbols stand for the data points associated with three lepton flavors $e, \mu$ and $\tau$, respectively. In the right panel, the integrated scalar amplitude as a function of $T_{\mathrm{box}}$ is shown. The exponentially growing contamination has been removed for the three lepton flavors. For a comparison, we also show the $\mu$-data, which still contains the exponentially growing contamination, by the red triangle-up symbol.

For the Type 1 diagram given in Fig. 1, the corresponding unintegrated scalar amplitude is shown in the left panel of Fig. 3. At the time region $t_{\Delta S=1} \ll t_{\Delta S=0}$, the intermediate state is dominated by the ground state, i.e. the single lepton state. Among the three lepton flavors $\ell=e, \mu, \tau$, we have observed the exponentially growing time dependence for $\mu$. This is consistent with our expectation since the muon mass is lighter than the initial kaon mass. For the $e$ flavor, the exponentially growing behavior does not appear due to the helicity suppression in the process of $K^{+} \rightarrow e^{+} v \rightarrow \pi^{+} v \bar{v}$. For the $\tau$ flavor, since the intermediate states are much heavier than the initial state, there are no exponentially growing contributions. We calculated the matrix elements $\left\langle\pi^{+} v \bar{v}\left|O_{u \ell}^{\Delta S=0}(0)\right| \ell^{+} v\right\rangle$ and $\left\langle\ell^{+} v\left|O_{u \ell}^{\Delta S=1}(0)\right| K^{+}\right\rangle$, which give the contribution for the ground inter- 
mediate state. Using these matrix elements as inputs, we are able to remove the exponentially growing contamination.

In our calculations, we use the method described by Eq. (5.5). Both the times $t_{\Delta S=1}$ and $t_{\Delta S=0}$ are integrated over an interval with a size $T_{\mathrm{box}}$. The integrated amplitude as a function of $T_{\text {box }}$ is shown in the right panel of Fig. 3. The red triangle-up data points show the amplitude for the $\mu$ flavor, which contains the exponentially growing contamination. The red square points show the same amplitude without exponential contamination. As we can see, after removing the contamination, the data can be described by a linear function. Through the fit, we determine the scalar amplitude $F_{W W}\left(p_{K}, p_{v}, p_{\bar{v}}\right)$ for three lepton flavors. The corresponding results are shown in Table 1. For a comparison, we use a model, which assumes only single-lepton contribution in the intermediate state, to estimate the scalar amplitude

$$
\text { model: } F_{W W}=-f_{K} f_{\pi} \frac{2 q^{2}}{q^{2}+m_{\ell}^{2}}, \quad q^{2}=\left(p_{K}-p_{v}\right)^{2},
$$

where $f_{\pi}$ and $f_{K}$ are pion and kaon decay constants. As shown in Table 1, the lattice results agree well with the model prediction. It suggests the ground-state dominance for the Type 1 diagram, and the excited-intermediate-state effects are very small $(\lesssim 3 \%)$.

\begin{tabular}{c|cc|c}
\hline \hline$F_{W W}$ & Type 1 & model & Type 2 \\
\hline$e$ & $-1.685(47) \times 10^{-2}$ & $-1.740(6) \times 10^{-2}$ & $1.123(17) \times 10^{-1}$ \\
$\mu$ & $-1.818(40) \times 10^{-2}$ & $-1.822(6) \times 10^{-2}$ & $1.194(18) \times 10^{-1}$ \\
$\tau$ & $1.491(36) \times 10^{-3}$ & $1.471(5) \times 10^{-3}$ & $4.690(77) \times 10^{-2}$ \\
\hline
\end{tabular}

Table 1: Lattice results for the scalar amplitude from the $W-W$ diagrams. All the results are shown in lattice units. The model values are compiled using the lattice QCD determination of $f_{\pi}=0.08904(19)$ and $f_{K}=0.09769(14)$ at $m_{\pi}=420 \mathrm{MeV}$.

Compared to the Type 1 diagram, the Type 2 diagram is more interesting. It is related to the GIM mechanism and the logarithmic SD divergence. The unintegrated scalar amplitude as a function of $t_{\Delta S=1}-t_{\Delta S=0}$ is shown in Fig. 4. By zooming into the plots, we can observe the exponentially growing time dependence for the $\mu$ flavor. Such time dependence is not very significant since now the ground intermediate state is given by a lepton together with a neutral pion $(420 \mathrm{MeV})$ and its energy is of a similar size as the initial kaon mass. Although small, such exponential contamination still contributes a sizeable systematic effect. We therefore calculate the matrix elements of $\left\langle\pi^{+} v \bar{v}\left|O_{u \ell}^{\Delta S=0}(0)\right| \pi^{0} \ell^{+} v\right\rangle$ and $\left\langle\pi^{0} \ell^{+} v\left|O_{u \ell}^{\Delta S=1}(0)\right| K^{+}\right\rangle$to remove this contamination. For the Type 2 diagram, we do not observe the exponentially growing behavior for the $e$ flavor. Usually there is no helicity suppression for the $e$ flavor, since at $t_{\Delta S=1} \ll t_{\Delta S=0}$ the Type 2 diagram is dominated by the semi-leptonic intermediate state, rather than the leptonic one. In our lattice calculations, we use the discrete lattice momenta $(2 \pi / L) \vec{n}$ for the intermediate hadronic particles. According to spatial momentum conservation, the momenta for the intermediate lepton are given by $-(2 \pi / L) \vec{n}-\vec{p}_{v}$. With this setup, for the ground intermediate state, the neutral pion carries zero momentum and the helicity suppression still takes effect for the $e$ flavor. This is the reason why the exponentially growing contamination disappears for the $e$ flavor. The method used to discretize the spatial momenta for the intermediate-state particles is not unique. It will introduce a power-law finite-size 


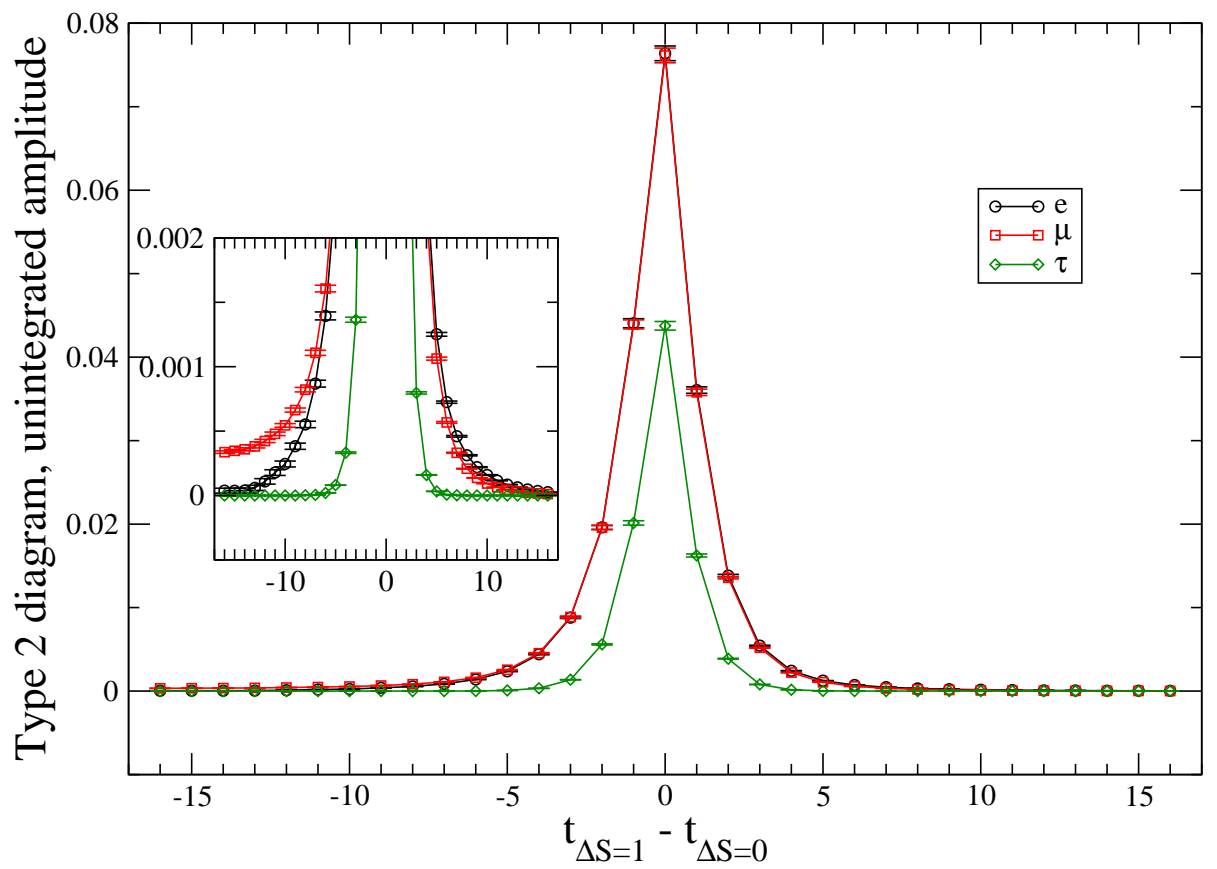

Figure 4: Unintegrated scalar amplitude for the Type 2 diagram.

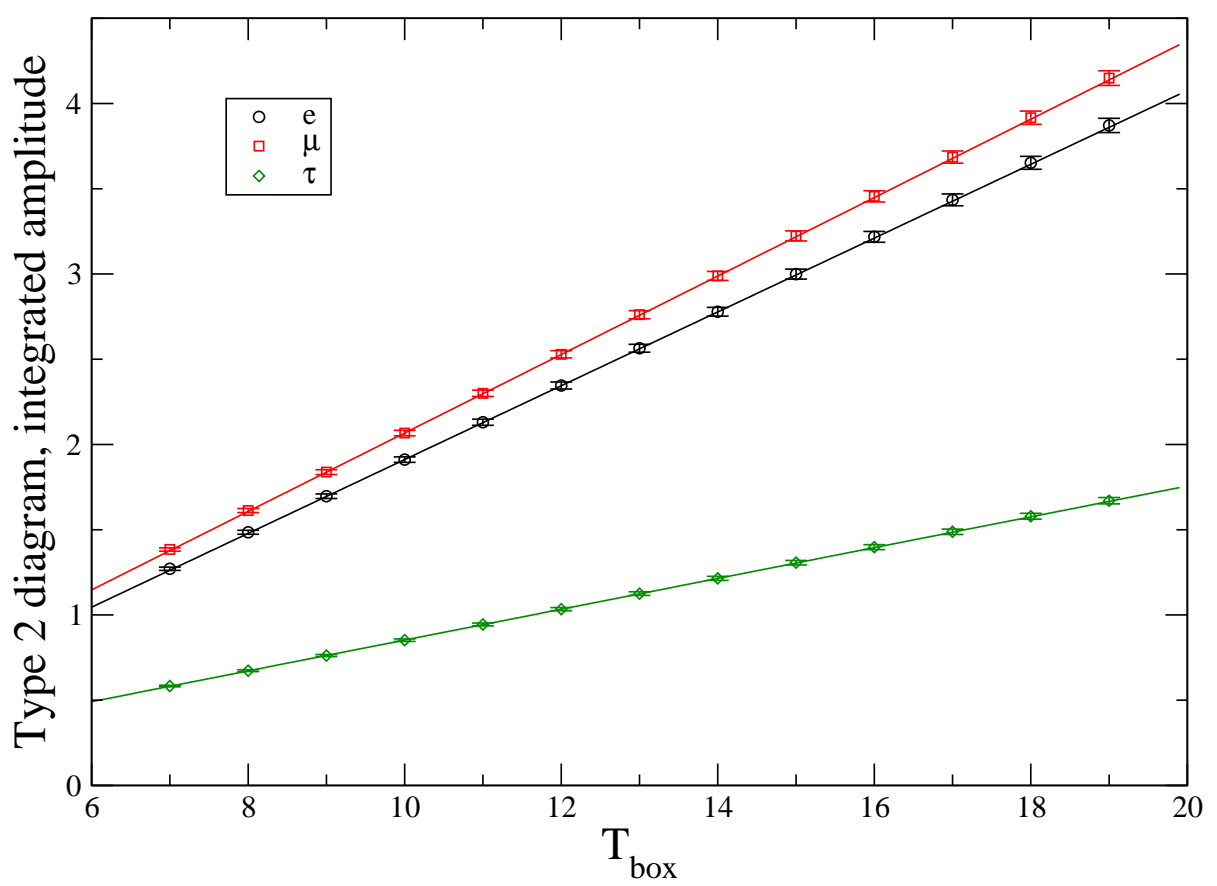

Figure 5: Integrated scalar amplitude for the Type 2 diagram. 
effect [28]. Controlling such finite-size effect is one of the research topics in our rare kaon decay project.

The integrated scalar amplitude for Type 2 diagram is shown in Fig. 5. By fitting the lattice data to a linear function of $T_{\mathrm{box}}$, we determine the values for $F_{W W}$ and list them in Table 1 . These values are much larger than the ones from Type 1 diagram. Note that the amplitude for Type 2 diagram contains a logarithmic SD divergence. This lattice cut-off SD piece must be removed by the counter term shown in Eq. (3.6) and replaced by the physical SD contribution.

\section{Numerical results for $Z$-exchange diagrams}

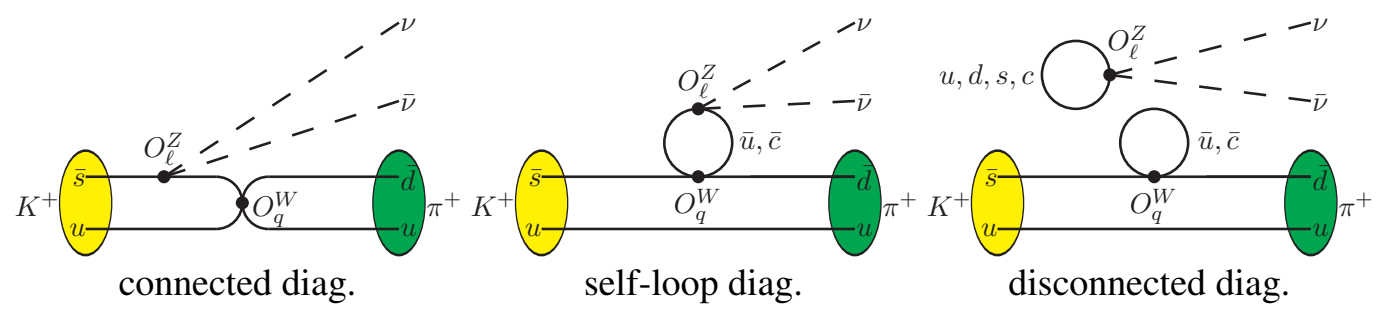

Figure 6: Samples of contractions contributing to $Z$-exchange diagrams. There are three different contraction structures: connected, self-loop and disconnected diagrams. For each of them we have picked up one example. A complete set of contractions can be found in our previous publication [20].

Samples of $Z$-exchange diagrams are given in Fig. 6. We write the bilocal matrix element in the form

$$
\begin{aligned}
T_{Z} & =\int d^{4} x\left\langle\pi^{+} v \bar{v}\left|T\left[O_{u}^{W}(x) O_{\ell}^{Z}(0)\right]\right| K^{+}\right\rangle-\{u \rightarrow c\} \\
& =T_{\mu}^{Z}\left[\bar{u}\left(p_{v}\right) \gamma_{\mu}\left(1-\gamma_{5}\right) v\left(p_{\bar{v}}\right)\right]
\end{aligned}
$$

where $O_{q}^{W}$ and $O_{\ell}^{Z}$ are defined in Eqs. (3.2) and (3.4). The hadronic part of $T_{Z}$ is given by

$$
T_{\mu}^{Z}=\int d^{4} x\left\langle\pi^{+}\left|T\left[O_{u}^{W}(x) J_{\mu}^{Z}(0)\right]\right| K^{+}\right\rangle-\{u \rightarrow c\} .
$$

We separate $T_{\mu}^{Z}$ into two parts: $T_{\mu}^{Z}=T_{\mu}^{Z, V}+T_{\mu}^{Z, A}$, corresponding to the vector $(V)$ and axial vector (A) components of $J_{\mu}^{Z}$. The $K \rightarrow \pi Z^{*}$ form factors can be obtained through

$$
T_{\mu}^{Z, i}=(+i)\left(F_{+}^{Z, i}\left(q^{2}\right)\left(p_{K}+p_{\pi}\right)_{\mu}+F_{-}^{Z, i}\left(q^{2}\right) q_{\mu}\right), \quad i=V, A,
$$

with $q=p_{K}-p_{\pi}$.

Since the spinor product $\bar{u}\left(p_{v}\right) q\left(1-\gamma_{5}\right) v\left(p_{\bar{v}}\right)$ vanishes under the assumption of massless neutrinos, only the form factors $F_{+}^{Z, i}\left(q^{2}\right)$ contribute to the decay amplitude. For the vector current, the Ward-Takahashi identity guarantees

$$
\left(m_{K}^{2}-m_{\pi}^{2}\right) F_{+}^{Z, V}\left(q^{2}\right)=q^{2} F_{-}^{Z, V}\left(q^{2}\right) .
$$

For the axial vector current, to separate $F_{+}^{Z, A}\left(q^{2}\right)$ from $T_{\mu}^{Z, A}$, we can compute the amplitude $T_{\mu}^{Z, A}$ for different $\mu$-polarization directions. This would require that either the kaon in the initial state or the pion in the final state carries non-zero spatial momentum. 
For the $Z$-exchange diagram a complete set of intermediate states can be inserted in-between $O_{u}^{W}$ and $J_{\mu}^{Z}$ in Eq. (6.2). We need to remove the exponentially growing contamination for those intermediate states whose energies are smaller than the initial kaon mass. For the vector current insertion, Ref. [20] shows that the parity-odd intermediate states, $\left|\pi^{+}\right\rangle$and $|3 \pi\rangle$, can cause exponentially growing contamination and need to be subtracted. For the axial vector current insertion, the parity-even state $|2 \pi\rangle$ will produce the exponentially growing contamination.

\subsection{Local vector current and short-distance divergence}

If one uses the conserved vector current, according to gauge invariance, one can write $T_{\mu}^{Z, V}\left(p_{K}, p_{\pi}\right)$ as

$$
T_{\mu}^{Z, V}\left(p_{K}, p_{\pi}\right)=(+i)\left(\frac{q^{2}}{m_{K}^{2}-m_{\pi}^{2}}\left(p_{K}+p_{\pi}\right)_{\mu}+q_{\mu}\right) F_{-}^{Z, V}\left(q^{2}\right) .
$$

The simplest momentum setup for the $K \rightarrow \pi Z^{*}$ transition is $\vec{p}_{K}=\vec{p}_{\pi}=\overrightarrow{0}$, where $\vec{p}_{K}$ and $\vec{p}_{\pi}$ are the spatial momenta for the kaon in the initial state and the pion in the final state. It appears that such a momentum choice is not very useful since the kinematic factor $\frac{q^{2}}{m_{K}^{2}-m_{\pi}^{2}}\left(p_{K}+p_{\pi}\right)_{\mu}+q_{\mu}$ is then equal to 0 . As a consequence, the transition amplitude $T_{\mu}^{Z, V}$ vanishes. However, if one uses the local vector current instead of the conserved one, then this momentum choice can be used to obtain a SD correction.

By using the local vector current, the Ward identity is violated and there will be a SD singularity when the operator $J_{\mu}^{Z, V}$ approaches the operator $O_{q}^{W}$. We must introduce a counter term $X(\bar{s} d)_{V-A}$ to correct this unphysical SD contribution which is cut off by the lattice spacing. Namely, we evaluate the bilocal matrix element together with a local matrix element

$$
\begin{aligned}
T_{\mu}^{Z, V}\left(p_{K}, p_{\pi}\right)= & Z_{V} \int d^{4} x\left\langle\pi^{+}\left|T\left[O_{u}^{W}(x) J_{\mu}^{V, l o c}(0)\right]\right| K^{+}\right\rangle-\{u \rightarrow c\} \\
& +X\left\langle\pi^{+}\right|\left(\bar{s} \gamma_{\mu}\left(1-\gamma_{5}\right) d(0)\left|K^{+}\right\rangle\right.
\end{aligned}
$$

where $J_{\mu}^{V, l o c}$ is a local vector current. For the LD contribution, we have $Z_{V} J_{\mu}^{V, l o c}=J_{\mu}^{V, c o n}$, where $Z_{V}$ is the vector current renormalization constant and $J_{\mu}^{V, c o n}$ is the conserved vector current. For the SD contribution, $X(\bar{s} d)_{V-A}$ has been introduced to correct the SD divergence in $O_{u}^{W}(x) J_{\mu}^{V, l o c}(0)$ at $x \rightarrow 0$. Here we need to determine the value for $X$. A natural condition we can use is $T_{\mu}^{Z, V}=0$ for $\vec{p}_{K}=\vec{p}_{\pi}=0$. We therefore compute the bilocal matrix element for two momentum choices: one has been discussed in Sect. 5.2; the other is $\vec{p}_{K}=\vec{p}_{\pi}=0$. The latter is used for the SD correction. Combining the two momentum choices together, we can determine the physical scalar amplitude for the $Z$-exchange diagram with the vector current insertion.

\subsection{Preliminary numerical results}

In this subsection, we first present the numerical results for the vector current insertion. The unintegrated matrix elements $\int d^{3} \vec{x}\left\langle\pi^{+}\left|T\left[H_{W}(\vec{x}, t) J_{\mu}^{V, l o c}(0)\right]\right| K^{+}\right\rangle$as a function of the time separation $t$ are shown in the upper panel of Fig. 7, where the Hamiltonian $H_{W}$ is given by $H_{W}=O_{u}^{W}-O_{c}^{W}$. Since the operator $O_{q}^{W}$ consists of $Q_{1, q}$ and $Q_{2, q}$ operators, we show the numerical results for both cases. The polarization direction for the vector current $J_{\mu}^{V, l o c}$ is chosen to be $\mu=t$. The black circle 

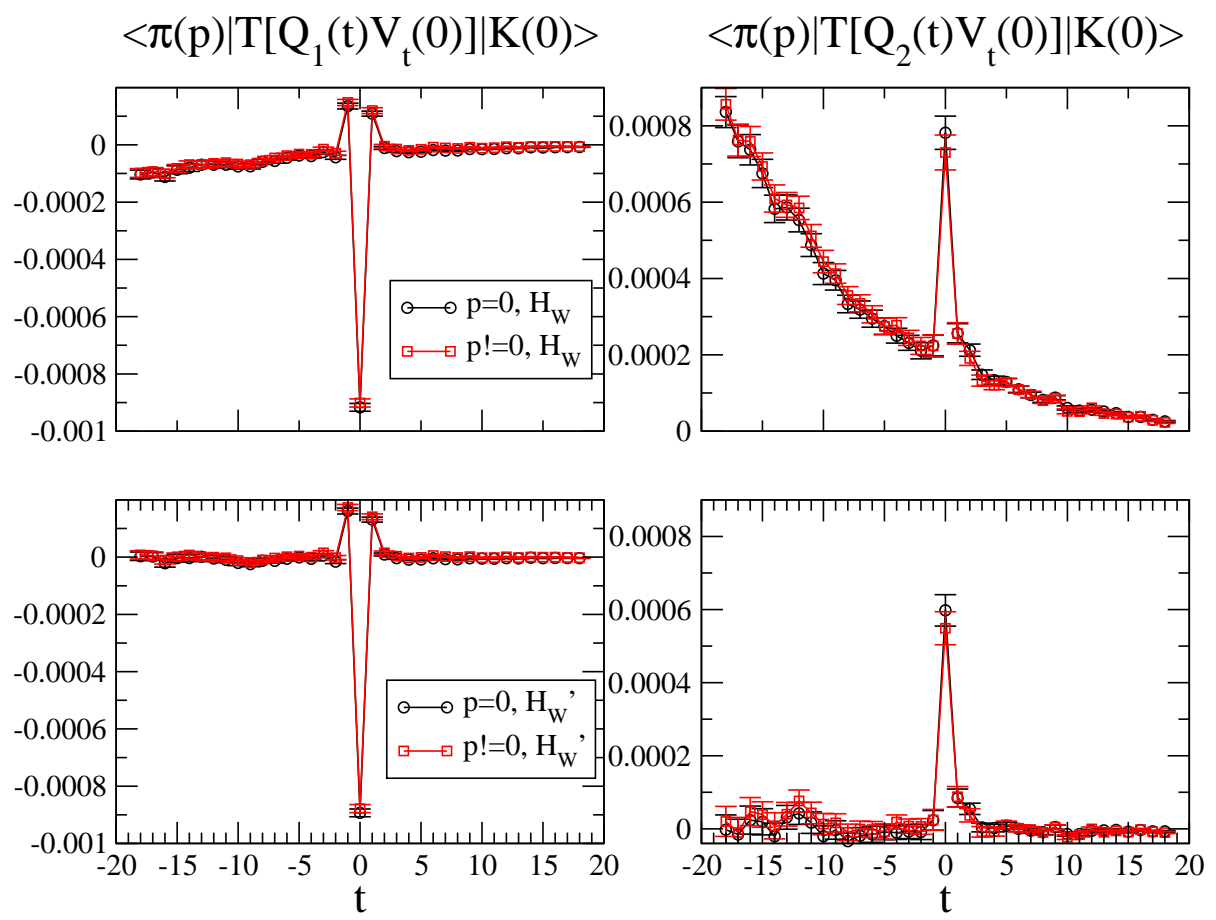

Figure 7: Unintegrated matrix elements for the $Z$-exchange diagram with the vector current insertion. The vector current polarization direction is chosen to be $\mu=t$. In the upper panel, the matrix elements $\int d^{3} \vec{x}\left\langle\pi^{+}\left(p_{\pi}\right)\right| T\left[H_{W}(\vec{x}, t) J_{\mu=t}^{V, l o c}(0)\left|K^{+}(0)\right\rangle\right.$ (for $Q_{1, q}$ and $Q_{2, q}$ components) as a function of $t$ is shown. The black circle data points show the lattice results for the momentum mode $\vec{p}_{K}=\vec{p}_{\pi}=\overrightarrow{0}$; the red square points show the results for $\vec{p}_{K}=0$ and $\vec{p}_{\pi} \neq 0$. The exponentially growing time dependence can be seen at $t \ll 0$. In the lower panel, the matrix elements are calculated using a new Hamiltonian $H_{W}^{\prime}=H_{W}+c_{s}(\bar{s} d)$, where the exponentially growing contamination has been removed.

data points show the lattice results for the momentum $\vec{p}_{K}=\vec{p}_{\pi}=\overrightarrow{0}$; the red square points show the results for $\vec{p}_{K}=0$ and $\vec{p}_{\pi} \neq 0$. At $m_{\pi}=420 \mathrm{MeV}$ and $m_{K}=540 \mathrm{MeV}$, the on-shell $K^{+} \rightarrow \pi^{+} v \bar{v}$ decay only allows the pion to carry a small momentum $(\lesssim 30 \%$ of the pion mass). Thus, the black circle and red square data points are very close to each other.

In the time region $t \ll 0$, the dominant intermediate state is the single $\pi^{+}$state. Since this state is lighter than the initial state, there is exponentially growing contamination as shown in the upper panel of Fig. 7. In addition to the method described in Sect. 5.3, here we adopt a second approach to remove the exponentially growing contamination. We add to the weak Hamiltonian $H_{W}$ a scalar density operator $c_{s}(\bar{s} d): H_{W}^{\prime}=H_{W}+c_{s}(\bar{s} d)$. Using the chiral Ward identities, one can prove that the scalar density operator cannot contribute to an on-shell matrix element $[16,17,20]$. One can choose an appropriate value for $c_{s}$ through the equation

$$
\left\langle\pi^{+}(0)\left|H_{W}(0)+c_{s}(\bar{s} d)(0)\right| K^{+}(0)\right\rangle=0 .
$$

In the lower panel of Fig. 7 we show the unintegrated matrix element with the new Hamiltonian $H_{W}^{\prime}$. By adding the scalar density term, the exponentially growing contamination is removed.

For the axial vector current insertion, the unintegrated matrix elements are shown in Fig. 8. At $t \ll 0$ the time dependence is dominated by the two-pion state, whose energy is larger than 
the initial kaon mass, since $m_{\pi}=420 \mathrm{MeV}$. Thus the contamination is exponentially suppressed. (When in the future we use the physical pion mass, the two-pion contamination will not be exponentially suppressed, but can be removed by adding a pseudo-scalar density operator $c_{p}\left(\bar{s} \gamma_{5} d\right)$ to $H_{W}$.) By comparing the label of the y-axis of Figs. 7 and 8, our numerical results show that the axial vector current contributions are much larger than the vector current contributions. Note that the $Z$-exchange diagram with the axial vector current insertion is logarithmically divergent. A SD correction is essential in our numerical study.

In addition to the connected and self-loop diagrams in Fig. 6, we also calculate the disconnected diagrams and attempt to produce results including all quark-contraction diagrams. As shown by the green diamond symbol in Fig. 8, the absolute size of disconnected diagrams is much smaller than the size of connected and self-loop diagrams. Although the disconnected diagrams have much larger relative statistical errors, they do not contribute a large uncertainty in the total decay amplitude. Thus a complete lattice QCD calculation including all the diagrams is practical.

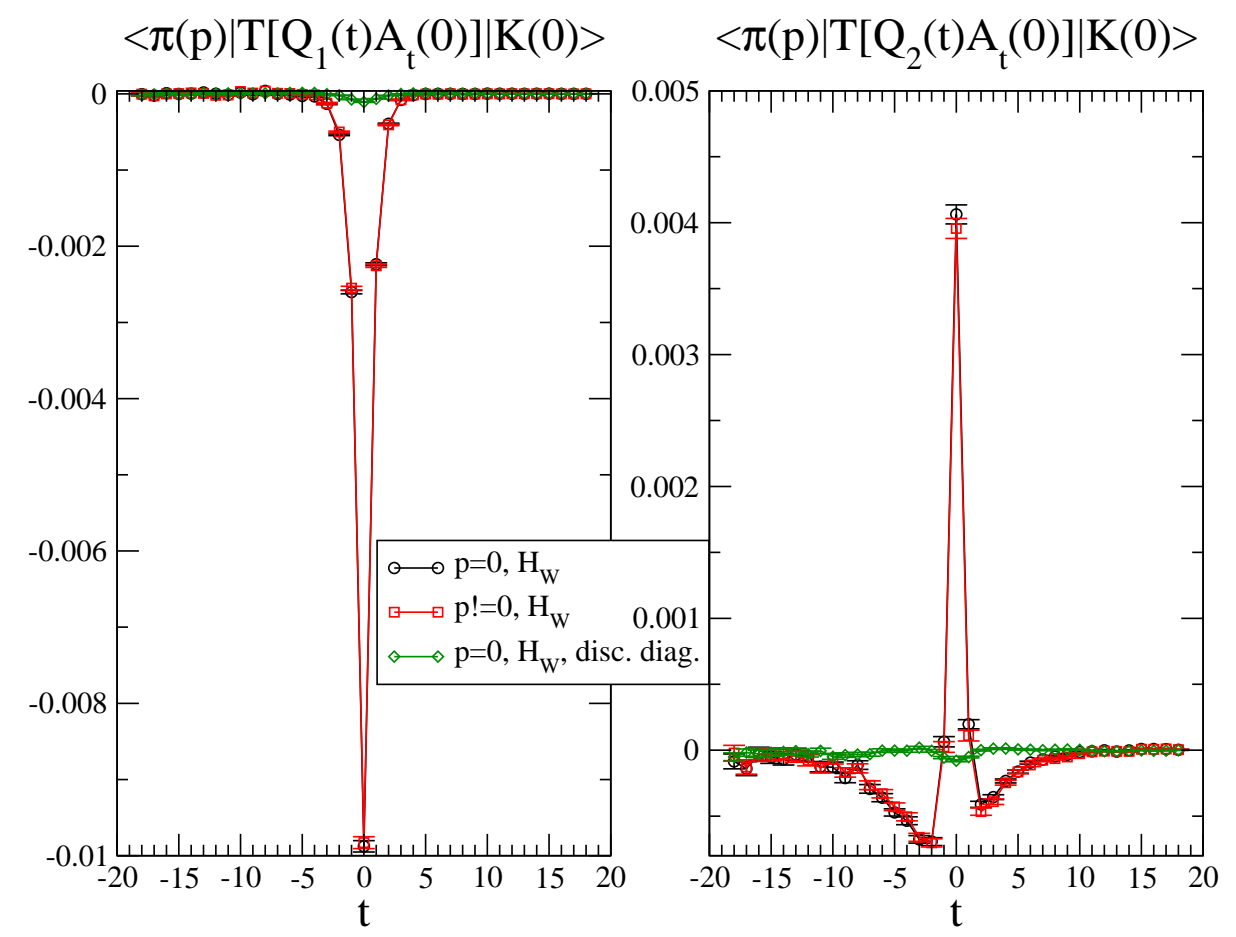

Figure 8: Unintegrated matrix elements for the $Z$-exchange diagram with the axial vector current insertion. The axial vector current polarization direction is chosen to be $\mu=t$. At $m_{\pi}=420 \mathrm{MeV}$, no exponentially growing contamination is observed at $t \ll 0$. The black circle data points show the lattice results for the momentum $\vec{p}_{K}=\vec{p}_{\pi}=\overrightarrow{0}$; the red square points show the results for $\vec{p}_{K}=0$ and $\vec{p}_{\pi} \neq 0$. These results include only the connected and self-loop diagrams. For the disconnected diagrams, the corresponding results are shown by the green diamond symbol. Although noisy, the disconnected contributions are much smaller than the connected ones.

\section{Conclusion}

Recognizing the fact that NA62 experiment at CERN is collecting data for $K^{+} \rightarrow \pi^{+} v \bar{v}$ and the KOTO experiment at J-PARC is designed to search for $K_{L} \rightarrow \pi^{0} v \bar{v}$, these two rare kaon decay 
modes become important tools, complementary to the large $E_{T}$ searches at the LHC, in searches for an understanding of physics beyond the SM. In both channels the decay amplitudes are dominated by SD contributions. For $K_{L}$ decay, the LD contribution can be safely neglected. For $K^{+}$decay, the LD effects are expected to be a few percent. Although the size is small, it now serves as the dominant theory uncertainty for the SM prediction of $\operatorname{Br}\left(K^{+} \rightarrow \pi^{+} v \bar{v}\right)$. It is therefore timely for lattice QCD to provide the LD contributions to $K^{+} \rightarrow \pi^{+} v \bar{v}$ with controlled uncertainties.

The calculation of the $K^{+} \rightarrow \pi^{+} v \bar{v}$ decay amplitude is highly non-trivial. It involves the calculation of 4-point correlation functions and bilocal matrix elements. To correctly reproduce the physical amplitude, we need to isolate and remove the exponentially growing contamination from the intermediate states whose energies are lower than kaon initial state. We also need to deal with the logarithmic divergence which results when the two operators in the bilocal matrix element come close to each other. The power-law finite volume effect is another important systematic error which we need to control.

Using the methods developed by Refs. [14, 15, 16, 17, 28], our exploratory study shows the feasibility to calculate the LD contributions to the rare kaon decay $K^{+} \rightarrow \pi^{+} v \bar{v}$ using lattice QCD. Although the calculation reported here is performed on a gauge configuration ensemble with unphysical pion and charm quark mass $\left(m_{\pi}=420 \mathrm{MeV}\right.$ and $\left.m_{c}^{\overline{\mathrm{MS}}}(2 \mathrm{GeV})=860 \mathrm{MeV}\right)$, we are now performing the calculation at a near-physical pion mass. The future plan is to include the physical charm quark mass as well. We expect that, in the near future, exa-scale computing will enable us to perform a complete calculation of the rare kaon decay amplitude with all the systematic uncertainties under control.

\section{References}

[1] G. Isidori, F. Mescia, and C. Smith, Nucl.Phys. B718, 319 (2005), hep-ph/0503107.

[2] A. J. Buras, D. Buttazzo, J. Girrbach-Noe, and R. Knegjens, (2015), 1503.02693.

[3] E949 Collaboration, A. Artamonov et al., Phys.Rev.Lett. 101, 191802 (2008), 0808.2459.

[4] E787 Collaboration, S. Adler et al., Phys.Rev.Lett. 79, 2204 (1997), hep-ex/9708031.

[5] E787 Collaboration, S. Adler et al., Phys.Rev.Lett. 84, 3768 (2000), hep-ex/0002015.

[6] E787 Collaboration, S. Adler et al., Phys.Rev.Lett. 88, 041803 (2002), hep-ex/0111091.

[7] E787 Collaboration, S. S. Adler et al., Phys.Lett. B537, 211 (2002), hep-ex/0201037.

[8] E949 Collaboration, V. Anisimovsky et al., Phys.Rev.Lett. 93, 031801 (2004), hep-ex/0403036.

[9] F. Newson et al., Prospects for $K^{+} \rightarrow \pi^{+} v \bar{v}$ at CERN in NA62, in 50 Years of CP Violation London, UK, July 10-11, 2014, 2014, 1411.0109.

[10] A. Romano, (2014), 1411.6546.

[11] T. K. Komatsubara, Prog. Part. Nucl. Phys. 67, 995 (2012), 1203.6437.

[12] КОТО, K. Shiomi, $K_{L}^{0} \rightarrow \pi^{0} v \bar{v}$ at KOTO, in 8th International Workshop on the CKM Unitarity Triangle (CKM2014) Vienna, Austria, September 8-12, 2014, 2014, 1411.4250.

[13] G. Isidori, G. Martinelli, and P. Turchetti, Phys.Lett. B633, 75 (2006), hep-lat/0506026.

[14] RBC Collaboration, UKQCD Collaboration, N. H. Christ, (2010), 1012.6034. 
[15] N. H. Christ, PoS LATTICE2011, 277 (2011), 1201.2065.

[16] RBC and UKQCD Collaborations, N. Christ, T. Izubuchi, C. Sachrajda, A. Soni, and J. Yu, Phys.Rev. D88, 014508 (2013), 1212.5931.

[17] Z. Bai et al., Phys.Rev.Lett. 113, 112003 (2014), 1406.0916.

[18] Z. Bai and N. H. Christ, PoS LATTICE2015, 342 (2016).

[19] X. Feng, N. H. Christ, A. Portelli, and C. Sachrajda, PoS LATTICE2014, 367 (2015).

[20] N. H. Christ, X. Feng, A. Portelli, and C. T. Sachrajda, (2015), 1507.03094.

[21] N. Christ et al., Long distance contributions to the rare kaon decay $K \rightarrow \pi \ell^{+} \ell^{-}$, in Proceedings, 33rd International Symposium on Lattice Field Theory (Lattice 2015), 2016, 1602.01374.

[22] V. Cirigliano, G. Ecker, H. Neufeld, A. Pich, and J. Portoles, Rev.Mod.Phys. 84, 399 (2012), 1107.6001 .

[23] A. Buras, M. Gorbahn, U. Haisch, and U. Nierste, Phys.Rev.Lett. 95, 261805 (2005), hep-ph/0508165.

[24] A. J. Buras, M. Gorbahn, U. Haisch, and U. Nierste, JHEP 0611, 002 (2006), hep-ph/0603079.

[25] G. Martinelli, C. Pittori, C. T. Sachrajda, M. Testa, and A. Vladikas, Nucl.Phys. B445, 81 (1995).

[26] RBC Collaboration, T. Blum et al., Phys.Rev. D68, 114506 (2003), hep-lat/0110075.

[27] C. Sturm et al., Phys. Rev. D80, 014501 (2009), 0901.2599.

[28] N. H. Christ, X. Feng, G. Martinelli, and C. T. Sachrajda, Phys. Rev. D91, 114510 (2015), 1504.01170. 